\title{
CERAS FOLIARES EPICUTICULARES DE ESPÉCIES CONGÊNERES DA MATA E DO CERRADO ${ }^{1}$
}

\author{
Elenice Mouro Varanda ${ }^{2}$ \\ Déborah Yara Alves Cursino dos Santos ${ }^{3}$
}

Recebido em 06.09.95. Aceito em 29.01.96.

RESUMO- (Ceras foliares epicuticulares de espécies congêneres da mata e do cerrado). Espécies de cerrado e mata foram analisadas quanto à sua composição em ceras foliares epicuticulares e de seus componentes hidrocarbonetos. Observou-se nas espécies de cerrado uma tendência a teores de ceras pouco maiores que os de espécies de mata estacional semidecídua. A porcentagem de hidrocarbonetos nas ceras foi maior na maioria das espécies de mata que nas espécies congêneres de cerrado. Pela análise em CG, os hidrocarbonetos mostraram predominância de $C_{29}$ e $C_{31}$ apresentando um comprimento médio da cadeia de carbono dos homólogos menos variável em espécies de mata, em torno de 30,5 , que de cerrado nas quais este valor variou de 28,5 a 31,3 . Os resultados são discutidos em relação ao provável papel ecológico das ceras e sua aplicação como marcadores taxônomicos.

Palavras chave: ceras epicuticulares; cerrado, floresta semidecídua; alcanos, ecologia.

\begin{abstract}
Foliar epicuticular waxes of congeneric species from forest and cerrado vegetation). Four woody species of cerrado and five woody species of seasonal semideciduous forest were analysed concerning the contents of epicuticular waxes and their parafinic profiles. The cerrado species showed a tendency to higher contents of epicuticular waxes and lower proportion of hydrocarbons than the forest species. The $\mathrm{C}_{29}$ and $\mathrm{C}_{31}$ alkanes were dominant in all species and the average lenght of the hydrocarbon chains were around 30,5 in the forest species and from 28,5 to 31,3 in the cerrado species. The ecological and taxonomic aspects of this characteristics are discussed.
\end{abstract}

Key words: epicuticular waxes; cerrado and semideciduous forest; alcanes, ecology.

\section{Introdução}

Todas as plantas terrestres são providas de uma camada hidrofóbica externa à camada cutinizada, de importância vital no sucesso adaptativo dos vegetais na

2 Departamento de Biologia, Faculdade de Filosofia, Ciências e Letras de Ribeirão Preto, USP, Av. Bandeirantes 3900, 14040-901, Ribeirão Preto - SP, Brasil.

${ }^{3}$ Departamento de Botânica, Instituto de Biociências, USP, Caixa Postal 11461, 05422-970, São Paulo SP, Brasil. 
conquista do ambiente terrestre, a cera epicuticular. As ceras são misturas complexas diferindo umas das outras em número, abundância relativa e distribuição dos homólogos constituintes de suas classes, sendo a dos álcoois primários uma das mais comuns. Os hidrocarbonetos constituem uma classe de grande ocorrência podendo representar uma alta proporção dos depósitos cerosos (Baker 1982).

Por sua localização entre a planta e o meio, as ceras epicuticulares participam de processos físicos e fisiológicos ecologicamente importantes, tais como controle da perda de água por transpiração (Martin \& Juniper 1970) e proteção contra radiação solar, principalmente contra os raios ultravioletas (Kreger 1958). Em um estudo feito com Cotyledon orbiculata, Robinson et al. (1993) sugeriram que a presença de depósitos cerosos espessos sobre a epiderme conferem uma fotoproteção muito eficiente a plantas expostas a ambientes com alta incidência luminosa. Vários trabalhos ressaltam também a participação das ceras como barreira ao ataque de fungos patogênicos e de herbívoros (Martin \& Juniper 1970; Eglinton \& Hamilton 1967). Estudos recentes mostram o efeito de repelente alimentar para formigas (saúvas) apresentado por ceras de algumas espécies arbóreas do cerrado (Sugayama \& Salatino 1995). Varanda et al. (1992) também detectaram efeito deterrente do ácido ursólico da cera de Jacaranda decurrens sobre Schizaphis graminum. Tal composto provocou também queda na taxa de sobrevivência, no índice de reprodução e na taxa de crescimento populacional dos afídeos.

Muitos autores têm salientado a importância taxonômica do estudo das ceras epicuticulares, principalmente no que diz respeito a sua composição parafínica (Salatino \& Salatino 1983, Salatino et al. 1989, Blatt et al. 1991, Zygadlo et al. 1992, Vioque et al. 1994).

A vegetação do cerrado foi considerada durante vários anos como xerófila. No final da década de 40 verificou-se que as espécies arbóreas do cerrado transpiravam livremente mesmo durante a estação seca, não sendo a água um fator limitante para as mesmas (Ferri 1944, Rachid 1947, Rawitscher 1948). A partir da década de 50 outros fatores como o oligotrofismo do solo (Arens 1958, Salatino 1993), os altos teores de alumínio (Goodland 1971) e as queimadas periódicas (Coutinho 1976), foram apontados como responsáveis por seu aspecto xeromórfico.

Com relação às ceras epicuticulares, Amaral et al. (1985) estudando espécies de dicotiledôneas do cerrado, observaram discreta correlação entre o teor de cera e a espessura da camada de compostos lipofílicos com a pilosidade, porém nenhuma relação entre as características da cera e o grau de escleromorfismo de suas folhas.

Este trabalho tem por objetivo verificar se ocorre alguma variação nos teores de ceras foliares epicuticulares e na composição dos hidrocarbonetos entre espécies relacionadas do cerrado e da mata, a fim de contribuir para o conhecimento do seu papel ecológico e/ou da sua importância como marcador taxonômico.

\section{Material e métodos}

Folhas secas ao ar foram submetidas a três extrações sucessivas por imersão de 10 segundos em clorofórmio (Silva-Fernandes 1965). Os extratos foram reunidos, 
filtrados e concentrados em rotaevaporador sob pressão reduzida. As ceras obtidas foram pesadas e o rendimento expresso em relação à área foliar $\left(\mathrm{mg} \cdot \mathrm{dm}^{-2}\right)$.

A composição total da cera bruta foi obtida através de cromatografia em camada delgada ao lado de padrões em placa de sílica gel G60, utilizando clorofórmio como fase móvel. A fração de hidrocarbonetos foi obtida pelo fracionamento da cera bruta por cromatografia em coluna de gel de sílica, sendo eluída com éter de petróleo. Outras misturas de solventes mais polares foram utilizadas na lavagem das colunas para a retirada dos outros componentes da cera.

Os hidrocarbonetos foram identificados por cromatografia gasosa (Cromatógrafo CG-37-D, coluna capilar $10 \mathrm{~m}$ x 0,25 m, OV 101), por comparação dos tempos de retenção com os de amostras autênticas (Barta \& Kómives 1984). O comprimento médio $(\mathrm{CM})$ das cadeias carbônicas foi calculado de maneira idêntica à determinação de médias em distribuições de freqüência.

\section{Resultados e discussão}

Os dados obtidos mostram um teor de cera epicuticular muito variável entre as espécies estudadas (Tabela 1). Entretanto, observa-se uma tendência de teores maiores nas espécies coletadas no cerrado comparadas aos seus pares congêneres da mata. Comparando-se as espécies dos gêneros $\operatorname{Styrax}\left(S\right.$. camporum - 5,35 mg. dm ${ }^{-2} ; S$. sieberi - $1,41 \mathrm{mg}$. dm ${ }^{-2}$ ) e Qualea $\left(Q\right.$. densiflora $-6,73 \mathrm{mg} . \mathrm{dm}^{-2} ; Q$. grandiflora $\mathrm{C}$ $3,75 \mathrm{mg} \cdot \mathrm{dm}^{-2} ; Q$. grandiflora $\mathrm{M}-1,44 \mathrm{mg}$. $\mathrm{dm}^{-2}$ ) este teor é cerca de 3,5 vezes maior nas espécies de cerrado. No gênero Terminalia, a espécie do cerrado ( $T$. argentea) apresentou uma quantidade de cera maior $\left(2,3 \mathrm{mg} . \mathrm{dm}^{-2}\right)$ que $T$. modesta $\left(0,73 \mathrm{mg} . \mathrm{dm}^{-}\right.$ $\left.{ }^{2}\right)$ e menor que $T$. brasiliensis $\left(4,05 \mathrm{mg}\right.$. $\left.\mathrm{dm}^{-2}\right)$, ambas pertencentes à mata. Em Rapanea, a espécie da mata apresentou um teor pouco maior que aquela de cerrado (1,26 mg. dm ${ }^{-2}$ para $R$. guianensis e $1,40 \mathrm{mg}$. dm $\mathrm{d}^{-2}$ para $R$. umbellata).

Os teores obtidos para as espécies de cerrado analizadas foram menores que aqueles obtidos por Amaral et al. (1985) analisando outras espécies de dicotiledôneas deste ambiente. A expressão da quantidade das ceras pode estar relacionada a vários fatores bióticos e abióticos. O teor de cera pode estar muito mais relacionado às características intrínsecas ligadas ao patrimônio genético de cada planta que às condições do ambiente (Bengston et al. 1978). Este pode ser o caso das espécies estudadas no presente trabalho.

Uma grande variação também pode ser observada na porcentagem da fração de alcanos nas várias espécies analisadas (Tabela 1). Os maiores valores observados foram encontrados nas espécies de Qualea, nas quais esta fração foi a predominante. Outras espécies também apresentaram teores de hidrocarbonetos que podem ser considerados altos, porém não se pode afirmar se esta é a fração predominante nestas espécies por não ter sido feita uma análise quantitativa das demais frações. Não foi observada qualquer relação entre o teor de cera apresentado pelas espécies e a porcentagem de hidrocarbonetos das mesmas. Tulloch (1978) verificou que em Poa ampla a presença de alcanos não foi majoritária, havendo uma maior concentração de 
hidroxi- $\beta$-dicetonas. Lutz \& Gülz (1985) verificaram que os hidrocarbonetos representavam a classe de menor expressão nas ceras de sete espécies alpinas.

Tabela I. Locais de coleta, tipo de vegetação, teor de cera bruta, porcentagem de alcanos e comprimento médio das cadeias carbônicas dos homólogos nas espécies analisadas.

\begin{tabular}{|c|c|c|c|c|c|}
\hline Espécie & Local de coleta & $\begin{array}{l}\text { Tipo de } \\
\text { vegetação }\end{array}$ & $\begin{array}{c}\text { Teor } \\
\left(\mathrm{mg} \cdot \mathrm{dm}^{-2}\right)\end{array}$ & $\%$ alcanos & $\mathrm{CM} \#$ \\
\hline \multicolumn{6}{|l|}{ COMBRETACEAE } \\
\hline Terminalia argentea Mart. \& Zucc. & Fz. Sta Carlota * & cerrado & 2,30 & 7,75 & 30,1 \\
\hline Terminalia brasiliensis Camb. & Fz. Sta Carlota & mata & 4,05 & 16,07 & 31,1 \\
\hline $\begin{array}{l}\text { Terminalia modesta Eichl. } \\
\text { MYRSINACEAE }\end{array}$ & Fz. Sta Carlota & mata & 0,73 & 26,60 & 25,8 \\
\hline Rapanea guianensis Aulb. & Fz. Canchim ** & cerrado & 1,26 & 30,65 & 29,8 \\
\hline $\begin{array}{l}\text { Rapanea umbellata (Mart.) Mez. } \\
\text { STYRACACEAE }\end{array}$ & Fz. Sta Carlota & mata & 1,40 & 29,06 & 30,1 \\
\hline Styrax camporum Pohl. & Fz. Canchim & cerrado & 5,35 & 1,62 & 31,1 \\
\hline $\begin{array}{l}\text { Styrax sieberi Perk. } \\
\text { VOCHYSIACEAE }\end{array}$ & Fz. Sta Carlota & mata & 1,41 & 26,47 & 30,5 \\
\hline Qualea densiflora Warm. & Fz. Sta Carlota & cerrado & 6,73 & 71,26 & 28,8 \\
\hline Qualea grandiflora Mart. & Fz. Sta Carlota & mata & 3,75 & 38,90 & 30,4 \\
\hline Qualea grandiflora Mart. & Fz. Campininha *** & cerrado & 1,44 & 69,90 & 30,5 \\
\hline
\end{tabular}

Apesar das porcentagens dos alcanos serem muito variáveis, parece existir uma tendência a valores maiores nas espécies de mata analisadas neste trabalho, excetuando $Q$. densiflora (cerrado) que exibiu o maior valor observado. Esta maior concentração de alcanos nas ceras de espécies de mata (ambiente com alta umidade) pode estar relacionada a características que promovam a repelência de água, visto estas substâncias proporcionarem um maior ângulo de contato $\left(107-108^{\circ}\right)$ na interface entre a superfície foliar e a água (Martin \& Juniper 1970, Juniper \& Jefree 1983). A ausência de água nas superfícies foliares proporciona uma barreira ao desenvolvimento de microorganismos e diminue a resistência à difusão dos gases.

Segundo Harborne \& Turner (1984) a razão entre homólogos de número ímpar de átomos de carbono e aqueles de número par freqüentemente ocorre na proporção de 10:1. A maioria das espécies analisadas apresentaram uma razão bem maior que esta mencionada acima (Figuras $1 \mathrm{e} 2$ ). A presença de constituintes de cadeias mais longas está relacionada a uma maior rigidez do órgão devido ao aumento do ponto de fusão da cera epicuticular, além de contribuir para a eficiência da barreira contra perda de água (Hadley 1981).

Comparando a composição de alcanos das espécies analisadas, aquelas da mata parecem apresentar uma pequena tendência a homólogos de maior comprimento de cadeia quando comparadas aos seus pares congenéricos do cerrado, excetuando o gênero Styrax, no qual $S$. camporum apresentou CM maior $(31,1)$ que seu par, $S$. 

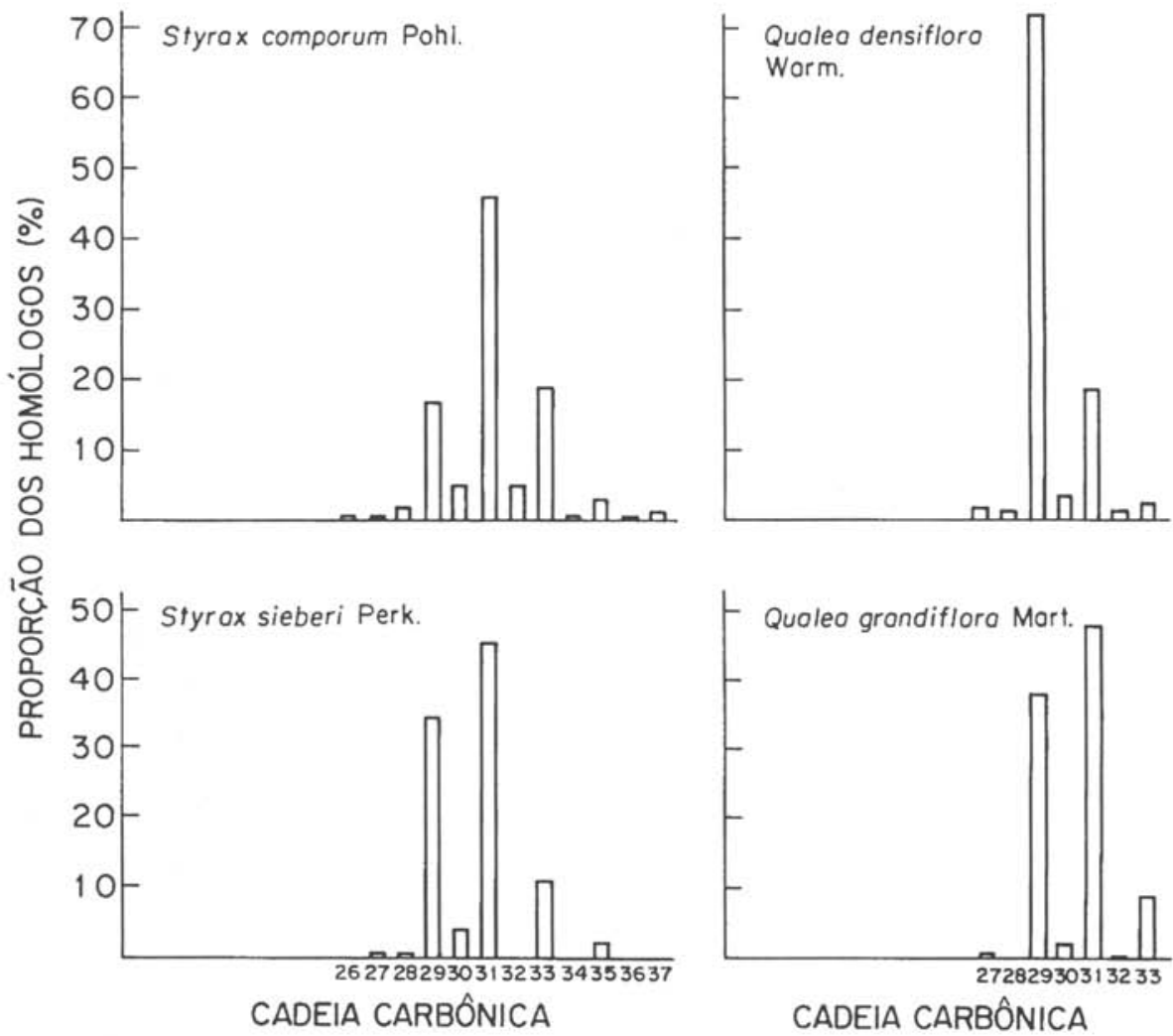

Figura 1. Composição da fração parafínica das ceras foliares epicuticulares de espécies de Styrax e Qualea.

sieberi $(30,5)$. Devido às características de escleromorfismo das espécies do cerrado seria esperado que estas apresentassem os maiores valores de comprimento médio das cadeias carbônicas quando comparadas às da mata.

Entre os pares de espécies estudadas foi observada uma grande similaridade quanto ao perfil dos homólogos de hidrocarbonetos presentes, com exceção daquelas do gênero Terminalia. Com isso, de maneira geral parece que fatores ambientais não têm grande influência na composição da fração dos hidrocarbonetos nas ceras epicuticulares. As espécies de Styrax (Figura 1) e Rapanea (Figura 2) apresentaram um predomínio de C29 e C31, além de uma maior expressão do homólogo C33. Em $Q$ ualea grandiflora o homólogo principal foi C31 enquanto que em $Q$. densiflora foi C29, que representou mais de 50\% do total dos hidrocarbonetos (Figura 1).

Dentre os indivíduos analisados, as maiores diferanças interespecíficas foram observadas nas espécies de Terminalia (Figura 2). Em $T$. argentea (cerrado) observou-se uma amplitude de composição dos hidrocarbonetos de C27 a C35, com predominância de $\mathrm{C} 29$ e C31, enquanto que $T$. brasiliensis apresentou uma distribui- 


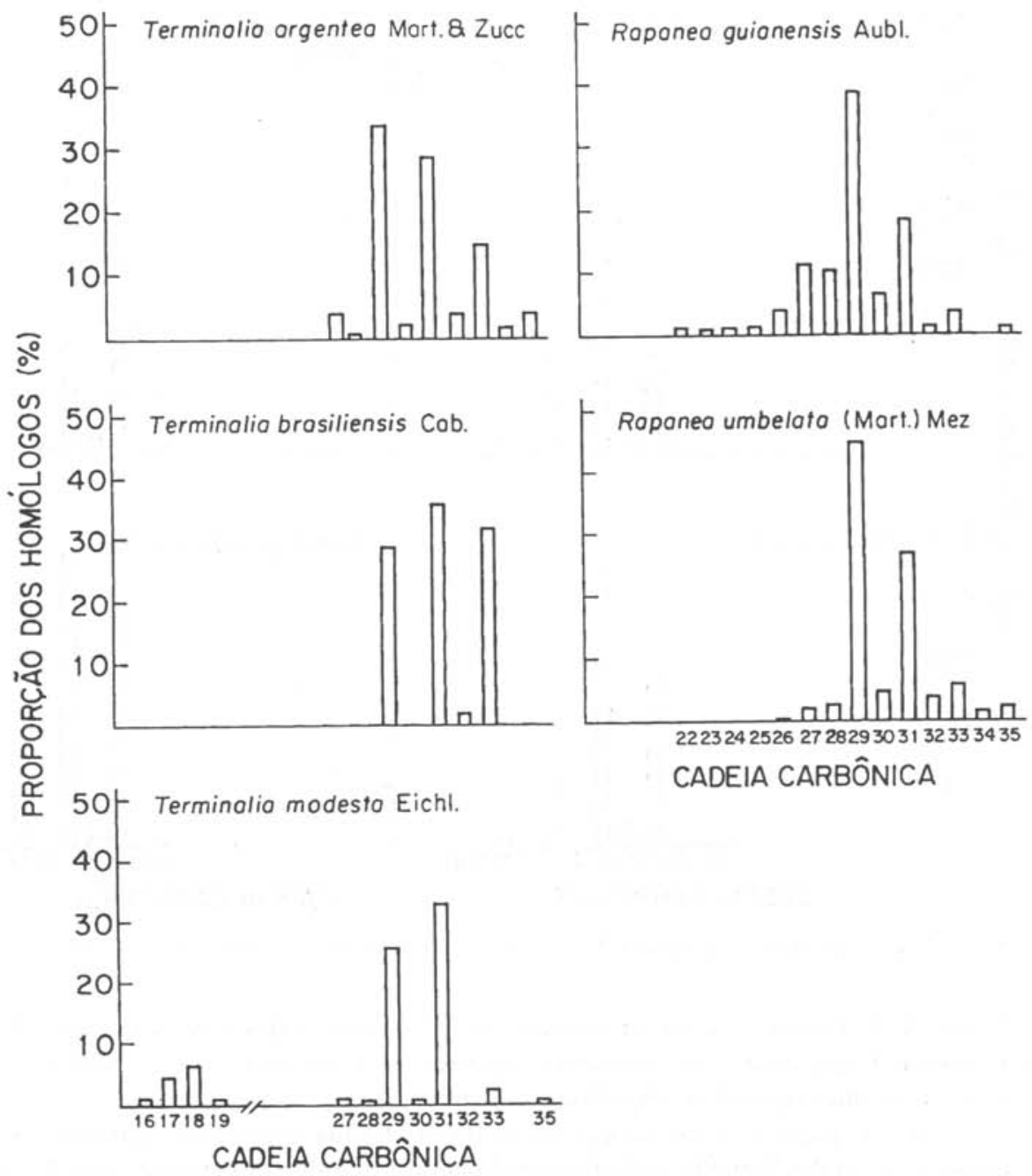

Figura 2. Composição da fração parafínica das ceras foliares epicuticulares de espécies de Terminalia e Rapanea.

ção mais restrita com acentuado predomínio de C29 e proporções elevadas de C31 e C33. T. modesta apresentou homólogos de cadeia curta (C16 a C19) não detectados nas duas espécies anteriores.

Os dados obtidos concordam com a literatura que afirma que os hidrocarbonetos apresentam predominantemente homólogos com número ímpar de átomos de carbono, entre os quais C29 e C31 são os mais abundantes (Eglinton et al. 1962, Eglinton \& Hamilton 1967, Douglas \& Eglinton 1966). 
Com os resultados obtidos conclui-se que dados provinientes das ceras foliares epicuticulares podem ser úteis como subsídio para estudos taxonômicos, principalmente com base na sua fração de alcanos, como já verificado por diversos autores, e que investigações posteriores envolvendo um maior número de pares de espécies dos dois ambientes pesquisados podem fornecer dados interessantes para o entendimento do papel das ceras na natureza.

\section{Referências bibliográficas}

Amaral, M. C. E.; Salatino, M. L. F. \& Salatino, A. 1985. Teor de cera foliar epicuticular de dicotiledôneas do cerrado. Revta bras. Bot. 8: 127-130.

Arens, K. 1958. O cerrado como vegetação oligotrófica. Bolm Fac. Filos. Cienc. e Letr.Univ. São Paulo Bot. 15: 69-77.

Baker, E. A. 1982. Chemistry and morphology of plant epicuticular waxes. In: Cuttler, D.F., Alvin, K.L., Price, C.E. (ed.). The plant cuticle. Academic Press, London.

Barta, I. C. \& Kómives, T. 1984. Gas chromatographic method for rapid analysis of epicuticular waxes composition of plants. J. Chromatog. 287: 438-441.

Blatt, C. T. T.; Salatino, M. L. F. \& Salatino, A. 1991. Taxonomic implications of the distribution of alkanes of the foliar epicuticular waxes of Diplusodon Pohl. (Lythraceae). Proceedings of the 13th Int. Symp. on Capillary Chromatography 1: 661-667.

Bengtson, C.; Larsson, S. \& Liljenberg, C. 1978. Effects of water stress on cuticular transpiration, rate and amount and composition of epicuticular wax in seedlings of six oat varieties. Physiol. Plant. 44: 319 324.

Coutinho, L. M. 1976. Contribuição ao conhecimento do papel ecológico das queimadas na floração de especies de cerrado. Tese de Livre-Docência, Instituto de Biociências da Universidade de São Paulo.

Douglas, A. G. \& Eglinton, G. 1966. The distribution of alkanes. In: Swain, T. (ed.). Comparative Phytochemistry. Academic Press, London.

Eglinton, G.; Hamilton, R. J., Raphael, R. A. \& Gonzalez, A. G. 1962. Hydrocarbon constituents of the wax coating of plant leaves: a taxonomic survey. Phytochemistry 1: 89-102.

Eglinton, G. \& Hamilton, R. J. 1967. Leaf epicuticular waxes. Science 156: 1322-1335.

Ferri, M. G. 1944. Transpiração de plantas permanentes dos cerrados. Bolm Fac. Filos. Ciênc. e Letr. Univ. São Paulo Bot. 41:159-224.

Goodland, R. J. A. 1971. Oligotrofismo e alumínio no cerrado. In: Ferri, M.G. (ed.). III Simpósio sobre o cerrado. EDUSP e Ed. Edgard Blucher, São Paulo.

Hadley, N. F. 1981. Cuticular lipids of terrestrial plants and arthropods: a comparison of their structure, composition, and waterproofing function. Biol. Rev. 56: 23-47.

Harborne, J. B. \& Turner, B. L. 1984. Plant chemosystematics. Academic Press, London.

Juniper, B. E. \& Jefree, C. E. 1983. Plant surfaces. Edward Arnold, London.

Kreger, D. R. 1958. Wax. In: Ruhland, W.E. (ed.). Enciclopedia of plant physiology: Vol. 10. Springer Verlag, Berlin.

Lutz, C. \& Gülz, P.G. 1985. Comparative analysis of epicuticular waxes from some high alpine plants species. Z. Naturforsch. 40: 599-605.

Martin, J. \& Juniper, B. E. 1970. The cuticles of plants. St Martin's Press, New York.

Rachid, M. 1947. Transpiração e sistemas subterrâneos da vegetação de verão de campos cerrados de Emas. Bolm Fac. Filos. Ciênc. e Letr. Univ. São Paulo Bot. 5: 1-35.

Rawitscher, F. 1948. The water economy of the vegetation of the "campos cerrados"in Southern Brazil. J. Ecol. 36: 237-268.

Robinson, S. A.; Lovelock, C. E. \& Osmond, C. B. 1993. Wax as a mechanism for protection against photoinhibition.- A study of Cotyledon orbiculata. Bot. Act. 106: 307-312. 
Salatino, A. 1993. Chemical ecology and the theory ofoligotrophic scleromorphism. Anais da Academia Brasileira de Ciências 65: 1-13.

Salatino, M. L. F. \& Salatino, A. 1983. Constituents of the unsaponifiable fraction of the foliar epicuticular wax and the systematics of Annonaceae. Revta Bras. Bot. 6: 23- 28.

Salatino, M. L. F.; Salatino, A.; Menezes, N. L. \& Mello-Silva, R. 1989. Alkanes of foliar epicuticular waxes of Velloziaceae. Phytochemistry 28: 1105-1114.

Silva-Fernandes, A. M. S. 1965. Chemical and physical studies on plant cuticles. Ann. Appl. Biol. 56: $297-$ 304.

Sugayama, R. L. \& Salatino, A. 1995. Influence of leaf epicuticular waxes from cerrado species on substrate selection by Atta sexdens rubropilosa. Entomologia Experimentalis et Applicata 74: 6369.

Tulloch, A. P. 1978. Epicuticular wax of Poa ampla leaves. Phytochemistry 17: 1613-1615.

Varanda, E. M.; Salatino, A.; Zúñiga, G. E.; Roque, A. \& Corcuera, L. J. 1992. Effect of ursolic acid from epicuticular waxes of Jacaranda decurrens on Schizaphis graminum. J. Nat. Prod. 55: 800-803.

Vioque, J.; Pastor, J. \& Vioque, E. 1994. Leaf wax alkanes in the genus Coincya. Phytochemistry 36: 349352.

Zygadlo, J. A.; Abburra, R. E.; Maestri, D. M. \& Guzman, C. A. 1992. Distribution of alkanes and fatty acids in the Condalia montana (Rhamnaceae) species complex. Pl. Syst. Evol. 179: 89-93. 